\title{
Similarity and Consistency Assessment of Three Major Online Drug-Drug Interaction Resources
}

\author{
Elpida Kontsioti ${ }^{1}$, Simon Maskell ${ }^{1}$, Amina Bensalem² ${ }^{2}$ Bhaskar Dutta ${ }^{3}$, and Munir \\ Pirmohamed $^{4}$ \\ ${ }^{1}$ University of Liverpool \\ ${ }^{2}$ Ceva Santé Animale \\ ${ }^{3}$ AstraZeneca Gaithersburg \\ ${ }^{4}$ University of Liverpool Institute of Translational Medicine
}

November 22, 2021

\begin{abstract}
AIM: To explore the level of agreement on drug-drug interaction (DDI) information listed in three major online drug information resources (DIRs) in terms of: (1) interacting drug pairs; (2) severity rating; (3) evidence rating and (4) clinical management recommendations. METHODS: We extracted DDI information from the British National Formulary (BNF), Thesaurus, and Micromedex. Following drug name normalisation, we estimated the overlap of the DIRs. We annotated clinical management recommendations either manually, where possible, or through application of a machine learning algorithm. RESULTS: The DIRs contained 51,481 (BNF), 38,037 (Thesaurus), and 65,446 (Micromedex) drug pairs involved in DDIs. The number of common DDIs across the three DIRs was 6,970 (13.54\% of BNF, $18.32 \%$ of Thesaurus, and $10.65 \%$ of Micromedex). Micromedex and Thesaurus overall showed higher levels of similarity in their severity ratings, while the BNF agreed more with Micromedex on the critical severity ratings and with Thesaurus on the least significant ones. Evidence rating agreement between BNF and Micromedex was generally poor. Variation in clinical management recommendations was also identified, with some categories (i.e. Monitor and Adjust dose) showing higher levels of agreement compared to others (i.e. Use with caution, Wash-out, Modify administration). CONCLUSIONS: There is considerable variation in the DDIs included in the examined DIRs, together with variability in categorisation of severity and clinical advice given. DDIs labelled as critical are more likely to appear in multiple DIRs. Such variability in information could have deleterious consequences for patient safety, and there is a need for harmonisation and standardisation.
\end{abstract}

\section{Hosted file}

ddi_db_comparison.pdf available at https://authorea.com/users/440544/articles/546357similarity-and-consistency-assessment-of-three-major-online-drug-drug-interaction-

resources 


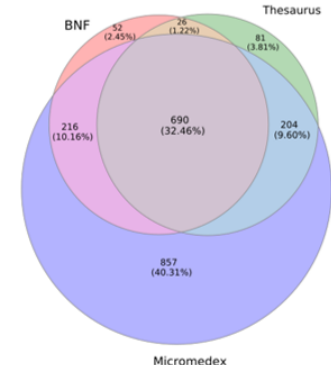

(a)

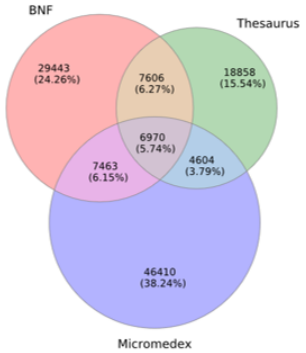

(b)

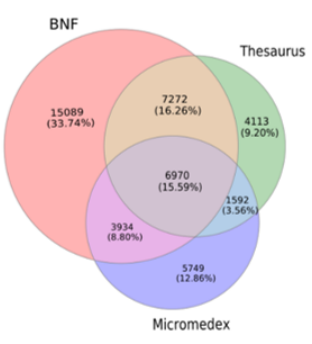

(c)

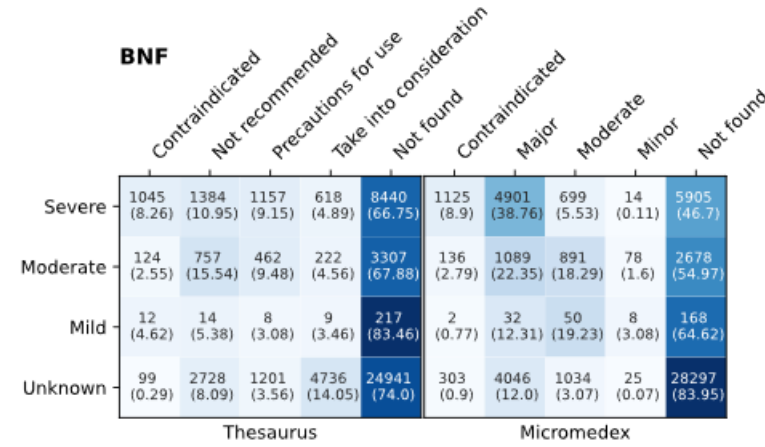

Thesaurus

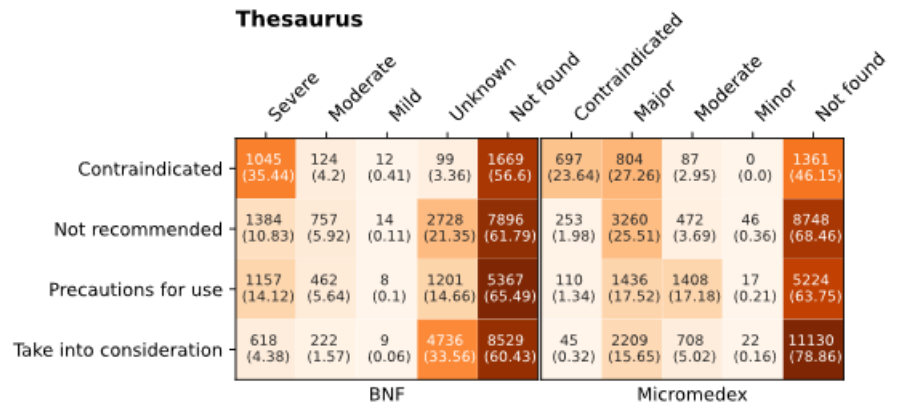

Micromedex
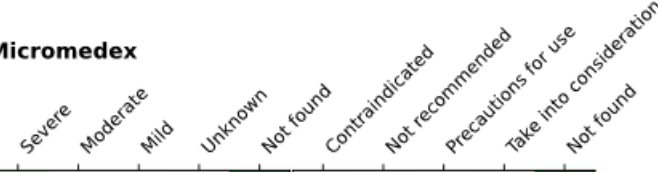

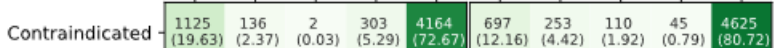

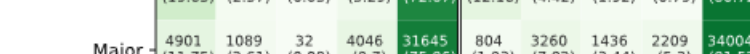

Major-f1901 \begin{tabular}{lllll|lllll}
4089 & 32 & 4046 & 31645 & 804 & 3260 & 1436 & 2209 & 34004 \\
$(11.75)$ & $(2.61)$ & $(0.08)$ & $(9.7)$ & $(75.86)$ & $(1.93)$ & $(7.82)$ & $(3.44)$ & $(5.3)$ & $(81.52)$
\end{tabular}

Moderate \begin{tabular}{lllll|l|llll|l}
699 & 891 & 50 & 1034 & 13216 & 87 & 472 & 1408 & 708 & 13215 \\
$(4.4)$ & $(5.61)$ & $(0.31)$ & $(6.51)$ & $(83.17)$ & $(0.55)$ & $(2.97)$ & $(8.86)$ & $(4.46)$ & $(83.17)$
\end{tabular}

Minor -\begin{tabular}{cccc|c|cccc|c}
14 & 78 & 8 & 25 & 1988 & 0 & 46 & 17 & 22 & 2028 \\
$(0.66)$ & $(3.69)$ & $(0.38)$ & $(1.18)$ & $(94.08)$ & $(0.0)$ & $(2.18)$ & $(0.8)$ & $(1.04)$ & $(95.98)$
\end{tabular}

BNF

Thesaurus 


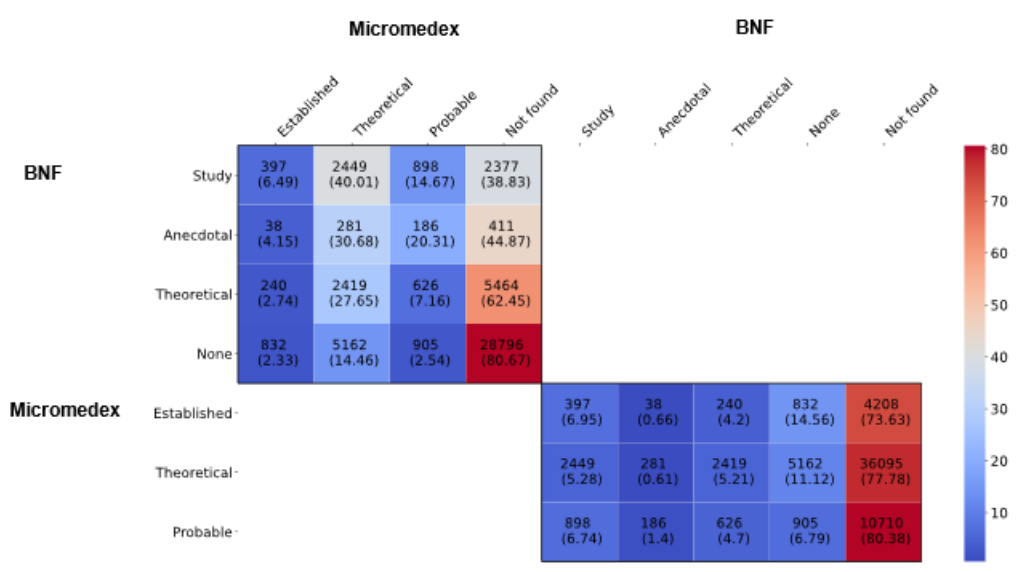

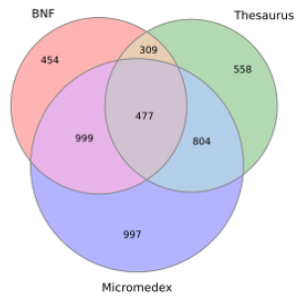

(a) Avoid
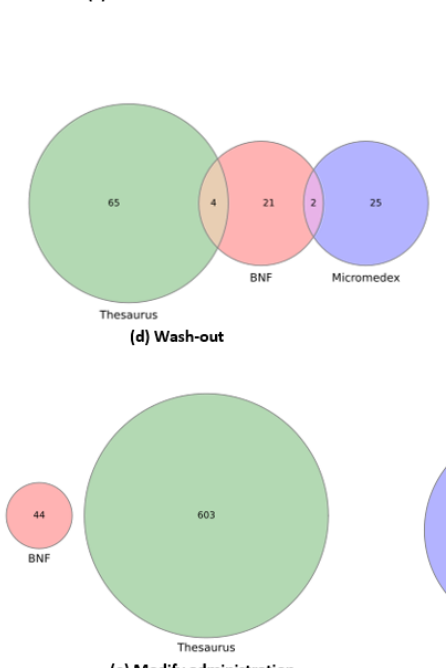

(e) Modify administration

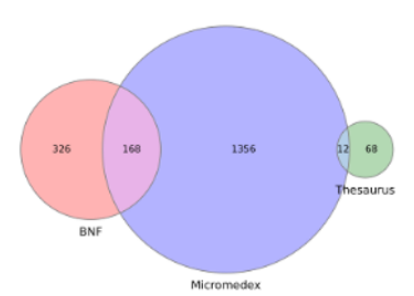

(b) Use with caution

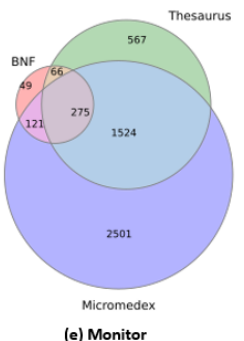

(e) Monitor

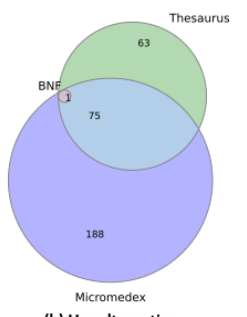

(h) Use alternative

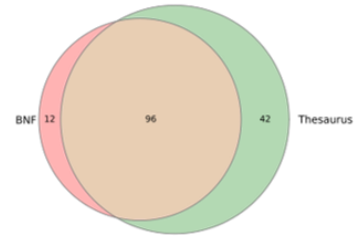

(c) Space dosing times
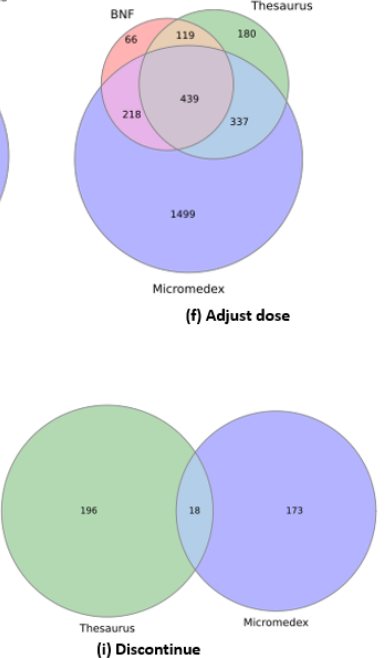